\title{
Adaptive Resource Scheduling Algorithm with QoS Guarantee in WiMAX Mesh Mode
}

\author{
http://dx.doi.org/10.3991/ijoe.v9i4.2823 \\ L.Y. Xiao, R. Wang, Z. Wang and H.N. Wang \\ China Organizational Name Administration Center, Beijing, China
}

\begin{abstract}
WiMAX is a new wireless metropolitan area networks technology based on IEEE 802.16 standard. WiMAX can provide high-speed connection for Internet. IEEE 802.16 standard is an open broadband wireless access technology, which supports high data rate and multimedia communications. Scheduling plays an important role in providing quality of service $(\mathrm{QoS})$ support to multimedia communications. IEEE 802.16e defined two topology architectures: point-to-multipoint and mesh. In this paper, an adaptive resource scheduling algorithm with QoS guarantees is proposed for WiMAX mesh mode. The scheduling order is determined according to the priority, QoS satisfaction and topology information of service flows in this algorithm. The system throughput, rtPS packet loss rate and nrtPS throughput of different algorithms are compared by simulation. Simulation results show that the proposed adaptive resource scheduling algorithm can meet various QoS requirements and increase the system throughput.
\end{abstract}

Index Terms-Mesh mode, QoS, resource scheduling, WiMAX

\section{INTRODUCTION}

IEEE802.16 standard, also known as WiMAX, is the one of the most important technologies for providing a Broadband Wireless Access(BWA) in a metropolitan area. With advantages of high transmission rate and predefined Quality-of-Service(QoS) framework, the IEEE802.16 standard has been developed to deliver a variety of multimedia applications with different QoS requirements. However, the IEEE802.16 standard only defines the QoS framework and signaling, but do not specify the scheduling algorithm that will ultimately provide QoS support. Scheduling algorithm is to make the users for sharing resources(available channels).

The medium access control(MAC) layer supports pointto-multipoint(PMP) architecture primarily, with an optional mesh topology. The main difference between the PMP and the mesh mode is that in the PMP mode traffic only occurs between the BS(base station) and SSs(Subscriber station), while in the mesh mode traffic can be routed through other SSs and can occur directly between SSs.

The resource scheduling algorithms about WiMAX are always based on the PMP mode. In this paper, the resource scheduling algorithm based on the mesh mode is given. We use graph coloring in the scheduling algorithm, which is a common method to solve resource allocation problems. The author T. H. Zheng proposed a network controlled spectrum access scheme where users behave collaboratively to optimize spectrum allocation for the entire network. The author developed a graph-theoretical model to characterize the spectrum access problem under a number of different optimization functions, and devise rules for users to utilize available spectrum while avoiding interference with its neighbors. In this paper, using the resource scheduling algorithms in PMP mode and the graph coloring, a new resource scheduling algorithms in mesh mode is proposed.

\section{RESOURCE SCHEDULING ALGORITHM}

\section{A. Service Type}

WiMAX defines five types of services: Unsolicited Grant Service(UGS), Real-time Polling Service(rtPS), Extended real-time Polling Service (ertPS), Non-realtime Polling Service(nrtPS) and Best Effort(BE). Different service type has different QoS requirement(such as the maximum latency, the minimum reserved data rate and so on ).

\section{B. Network Topology and Definition}

Fig.1 illustrates the network topology. There are $\mathrm{N}$ vertexes in the network topology, each vertex denotes a pair of transmitter and receiver, $M$ channels can be used during scheduling time. $\mathrm{N}$ vertexes share the $\mathrm{M}$ channels, so two vertexes within certain distance of each other can conflict if using same channel. In network topology, two vertexes connected with each other cannot use the same channel. We assume that environmental conditions such as vertex condition, available channel are static during channel allocation process.

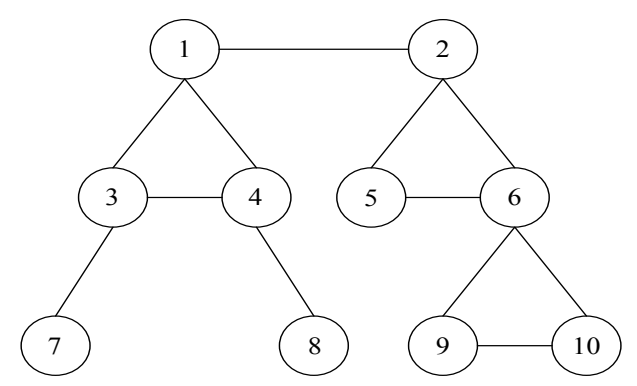

Figure 1. Network topology

The essence of channel allocation is to find an appropriate distribution of channels among vertexes so that they can coexist. In this paper, we assume the distribution is based on the service type, QoS requirement and the network topology.

Variables are defined as follows: 
- We assume scheduling cycle as the frame length.

- At beginning of each scheduling cycle, there are $N$ vertexes indexed from 1 to $N$ competing for $M$ channels indexed from 1 to $M$.

- Let $\mathbf{L}=\left\{l_{n, m} \mid l_{n, m} \in(0,1)\right\}_{N \times M}$ denote available channels for each vertex. The channel $m$ is available for vertex $n$ if $l_{n, m}=1$. $\sum_{m} l_{n, m}$ denotes the number of available channels for vertex $n$. Due to difference in service type, QoS requirement and the network topology, different vertex can get different available channels.

- Let $p_{i, n}$ express the length of packet $i$ that belongs to vertex $n$. $i$ express the packet's type, $i=1,2,3$ show that the packet $i$ belongs to rtPS, nrtPS or BE respectively.

- Let $R=\left\{r_{n, m}\right\}_{N \times M}$ denote the reward that vertex $n$ gets by acquiring available channel $m$ successfully. $r_{n, m}$ represents the maximum bandwidth/throughout that can be acquired (assuming no interference from other neighbors). In this paper, we assume the vertex can get the same reward in each channel, so $r_{n, m}$ can be simplified as $r$.

- Let $d_{i, n}$ express the number of channels to deliver the packet $i$ that belongs to vertex $n, d_{i, n}=\left\lceil\frac{p_{i, n}}{r}\right\rceil$.

- We use $q_{i, n}$ to express the queue length of traffic

flow with $i$ type that belong to vertex $n$. If $q_{i, n}=0$ , that means there is no packet to be delivered of connection $j$.

- We characterize interference between two competing vertexes by a constraint set. Let $\mathbf{C}=\left\{c_{n, k, m} \mid c_{n, k, m} \in\{0,1\}\right\}_{N \times N \times M}$ represent the interference constraint, where if $c_{n, k, m}=1$, vertex $n$ and $k$ would cause interference if they use the channel $m$ simultaneously.

- We define a valid channel assignment $\mathbf{A}=\left\{a_{n, m} \mid a_{n, m} \in\{0,1\}\right\}_{N \times M}$, where $a_{n, m}=1$ denotes the channel $m$ is allocated to vertex $n$. A satisfies all the constraints defined by $\mathbf{C}$, that is, $a_{n, m} \cdot a_{k, m}=0$, if $c_{n, k, m}=1, \forall n, k \in \mathrm{N}, m \in \mathrm{M}$.

\section{Optimization Problem}

The objective of the channel allocation is to maximize the overall system throughput while guaranteeing the QoS requirement, which is formulated into the following constrained optimization problem:

subject to

$$
\max \sum_{n=1}^{N} \sum_{m=1}^{M} a_{n, m} r_{n, m}
$$

$$
\begin{gathered}
a_{n, m} \cdot a_{k, m}=0, \text { if } c_{n, k, m}=1, \forall n, k \in \mathrm{N}, m \in \mathrm{M} \\
0 \leq \sum_{m=1}^{M} a_{n, m} \leq M \\
W_{k} \leq T_{r t P S}, \quad \forall k \in \Omega_{r t P S}
\end{gathered}
$$

$$
\bar{r}_{k} \geq r_{n r t P S}, \quad \forall k \in \Omega_{n r t P S}
$$

$\Omega_{r t P S}$ and $\Omega_{n r t P S}$ express the rtPS packet set and nrtPS packet set separately. (4) and (5) correspond to the Qos requirements in terms of delay and transmission rate. Here $T_{r t P S}$ and $r_{r t P S}$ are the maximum latency of rtPS type and minimum reserved rate of nrtPS type respectively.

The optimization problem above is hard to solve since there are many parameters that should be taken into account which render the implementation very complicated. So we give a feasible scheme.

\section{Adaptive Resource Scheduling Algorithm:}

First, we design the priority function of different service type.

For each rtPS packet, we define $\Phi_{i, n, j}$, its priority function as:

$$
\begin{gathered}
\Phi_{i, n, j}=\left\{\begin{array}{l}
\beta_{r t P S} \frac{1}{D_{n}+1} \frac{1}{F_{i, n, j}}, \quad \text { if } \quad F_{i, n, j} \geq 1 \\
\beta_{r t P S}, \quad \text { if } \quad F_{i, n, j}<1
\end{array}\right. \\
F_{i, n, j}=\frac{T_{r t P S}-W_{i, n, j}}{T_{g}}
\end{gathered}
$$

where $\beta_{r t P S} \in[0,1]$ is the rtPS-type coefficient, $D_{n}$ is the number of vertexes which are conflict with vertex $n$ and the $F_{i, n, j}$ is the delay satisfaction indicator, which is defined as the ratio of waiting time packet $i$ can continue enduring to the guard time. Where $W_{i, n, j}$ denotes the packet waiting time and $T_{g}$ is the guard time. Usually $T_{g}$ is set as frame length. If $F_{i, n, j}<1$, the packet $i$ should be sent immediately to avoid packet drop due to delay outage, so $\Phi_{i, n, j}$ is set to a high value $\beta_{r t P S}$. If $F_{i, n, j} \geq 1$ , the delay requirement is satisfied, and the priority function is quantified as $\beta_{r t P S} \frac{1}{D_{n}+1} \frac{1}{F_{i, n, j}}$. Large value of $F_{i, n, j}$ indicate high degree of satisfaction, which leads to low priority.

For each nrtPS packet, we define $\Phi_{i, n, j}$, its priority function as:

$$
\begin{aligned}
& \Phi_{i, n, j}= \begin{cases}\beta_{n r t P S} \frac{1}{D_{n}+1} \frac{1}{F_{i, n, j}}, & \text { if } F_{i, n, j} \geq 1 \\
\beta_{n r t P S}, & \text { if } \quad F_{i, n, j}<1\end{cases} \\
& F_{i, n, j}=\frac{\bar{r}_{i, n, j}(t)}{r_{n r t P S}} \\
& \bar{r}_{i, n, j}(t+1)=\bar{r}_{i, n . j}(t)\left(1-1 / t_{c}\right)+r_{i, n . j}(t) / t_{c}
\end{aligned}
$$

where $\beta_{n r t P S} \in[0,1]$ is the nrtPS-type coefficient and the $F_{i, n, j}$ is the rate satisfaction indicator, which is defined as the ratio of average transmission rate of packet $i$ to the minimum reserved data rate. The average transmission rate is estimated over a window $t_{c}$. If $F_{i, n, j}<1$, the packet $i$ 's average transmission rate is less than minimum reserved data rate, $\Phi_{i, n, j}$ is updated to a high value $\beta_{n r t P S}$. If $F_{i, n, j} \geq 1$, the rate requirement is 


\section{Adaptive Resource Scheduling Algorithm with QOS GuARANTEE IN WiMAX Mesh Mode}

satisfied, and the priority function is quantified as $\beta_{n r t P S} \frac{1}{D_{n}+1} \frac{1}{F_{i, n, j}}$. Large value of $F_{i, n, j}$ indicate high degree of satisfaction, which leads to low priority.

For each BE packet, we define $\Phi_{i, n, j}$, its priority function as:

$$
\Phi_{i, n, j}=\beta_{B E} \frac{1}{D_{n}+1}
$$

where $\beta_{B E} \in[0,1]$ is the BE-type coefficient. Since no QoS requirement is for $\mathrm{BE}$ type, the priority function is equal to $\beta_{B E} \frac{1}{D_{n}+1}$.

In each round of scheduling process, the scheduling order is based on the $\Phi_{i, n, j}$.

At the beginning of scheduling time $t$, we initialize the parameters, e.g. $l_{n, m}=1, a_{n, m}=0, \forall n \in \mathrm{N}, m \in \mathrm{M}$.

Step 1: Update $q_{i, n}$. We calculate the $\sum_{m} l_{n, m}$ for the vertex $n$ if it has packet to deliver(it means $q_{i, n} \neq 0$ ). If there exist $\sum_{m} l_{n, m} \neq 0$, go to step 2, else, go to step 5 .

Step 2: Calculate $\Phi_{i, n, j}$ of the packets based on equation (6), (8) and (11).

Step 3: Find out the packet that has largest $\Phi_{i, n, j}$, the length of the packet is $p_{i, n}$. Then, calculate the number of channels to deliver this packet:

$$
d_{i, n}=\left\lceil\frac{p_{i, n}}{r}\right\rceil
$$

If $d_{i, n} \leq \sum_{m} l_{n, m}$, give $d_{i, n}$ channels to the packet, go to step 4. If $d_{i, n}>\sum_{m} l_{n, m}, \operatorname{set} q_{j, n}=0$, go to step 1 .

Step 4: Update the parameters of vertex n, e.g. $l_{n, m}$, $a_{n, m}$ and the other vertexes' $l_{n, m}$ which are conflicted with vertex $n$, and then go to step 1 .

Step 5: Transmit the packets based on the scheduling results and the scheduling cycle is finished.

\section{COMPARATIVE ALGORITHMS}

\section{A. Graph coloring algorithm:}

In each scheduling time, the scheduling order is based on the $\Phi_{i, n, j}$.

$$
\Phi_{i, n, j}=\frac{1}{D_{n}+1}
$$

where $D_{n}$ is the number of vertexes which are conflict with vertex $n$. From equation (13), we can see that the packets of different type belongs to the same vertex have the same priority function. We suppose that the packets belong to the same vertex have a fixed scheduling order, that is rtPS, nrtPS and BE.

\section{B. Priority algorithm:}

First, define the priority function of different service type.

For rtPS packet, $\Phi_{i, n, j}$ is defined as follows:

$$
\begin{gathered}
\Phi_{i, n, j}= \begin{cases}\beta_{r t P S} \frac{1}{F_{i, n, j}}, & \text { if } F_{i, n, j} \geq 1 \\
\beta_{r t P S}, & \text { if } F_{i, n, j}<1\end{cases} \\
F_{i, n, j}=\frac{T_{r t P S}-W_{i, n, j}}{T_{g}}
\end{gathered}
$$

For nrtPS packet, $\Phi_{i, n, j}$ is defined as follows:

$$
\begin{gathered}
\Phi_{i, n, j}=\left\{\begin{array}{l}
\beta_{n r t P S} \frac{1}{F_{i, n, j}}, \quad \text { if } \quad F_{i, n, j} \geq 1 \\
\beta_{n r t P S}, \quad \text { if } F_{i, n, j}<1
\end{array}\right. \\
F_{i, n, j}=\frac{\bar{r}_{i, n, j}(t)}{r_{n r t P S}} \\
\bar{r}_{i, n, j}(t+1)=\bar{r}_{i, n, j}(t)\left(1-1 / t_{c}\right)+r_{i, n, j}(t) / t_{c}
\end{gathered}
$$

For BE packet, $\Phi_{i, n, j}$ is defined as follows:

$$
\Phi_{i, n, j}=\beta_{B E}
$$

The meanings of letters in function (14) to function (19) are same as described in section II. In each round of scheduling process, the scheduling order is based on the value of $\Phi_{i, n, j}$.

\section{SIMULATION RESULTS}

In the simulation, we assume there are 10 vertexes in the system and each has rtPS, nrtPS and BE connections respectively. The system generates 100 network topologies of 10 vertexes randomly.

Here we define length of packet is 1000 bits, and the coefficient of each type of service as: $\beta_{r t P S}=1$, $\beta_{n r t P S}=0.8, \beta_{B E}=0.6$. The length of frame is set to $5 \mathrm{~ms}$ that is the scheduling cycle. The simulation time is $100 \mathrm{~s}$, and $t_{c}$ is $1000 \mathrm{~ms}$. We suppose the packet arrival process is Poisson distributed, each connection with its own traffic rate as in Table I. Table I gives the maximum latency of rtPS packet and minimum reserved rate of nrtPS packet. We set the minimum reserved rate to be $85 \%$ of average traffic rate as usually defined in the standard. The channel rate is $200 \mathrm{~kb} / \mathrm{s}$.

TABLE I. SERVICE PARAMETERS

\begin{tabular}{|c|c|c|c|c|}
\hline \multicolumn{2}{|c|}{ rtPS } & \multicolumn{2}{c|}{ nrtPS } & BE \\
\hline $\begin{array}{c}\text { traffic } \\
\text { rate } \\
\left(\mathrm{kb} \cdot \mathrm{s}^{-1}\right)\end{array}$ & $\begin{array}{c}\text { maximum } \\
\text { latency } \\
(\mathrm{ms})\end{array}$ & $\begin{array}{c}\text { traffic } \\
\text { rate } \\
\left(\mathrm{kb} \cdot \mathrm{s}^{-1}\right)\end{array}$ & $\begin{array}{c}\text { minimum } \\
\text { reserved } \\
\text { rate } \\
\left(\mathrm{kb} \cdot \mathrm{s}^{-1}\right)\end{array}$ & $\begin{array}{c}\text { traffic } \\
\text { rate } \\
\left(\mathrm{kb} \cdot \mathrm{s}^{-1}\right)\end{array}$ \\
\hline $20-200$ & 30 & $40-400$ & $34-340$ & $80-800$ \\
\hline
\end{tabular}

In fig. 2, we compare the system throughput under our scheduling algorithm with graph coloring algorithm and priority algorithm. It can be observed that the system throughput increase as the total traffic rate changes for the three algorithms. When the total traffic rate is small, the system throughputs of the three algorithms are same and equal to the total traffic rate. This means that in the 
simulation process all the packet are sent successfully. When the total traffic rate is large, the graph coloring algorithm achieves the highest system throughput than other algorithms. And adaptive algorithm's system throughput is higher than priority algorithm's system throughput.

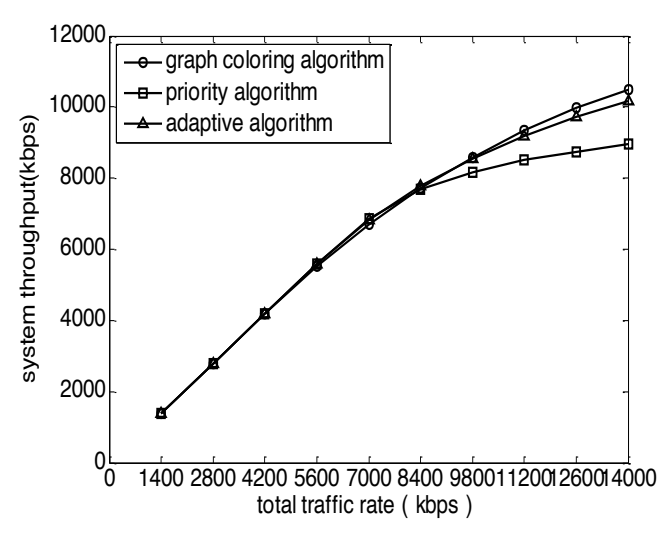

Figure 2. System throughput versus total traffic rate

Fig. 3 show the variation for packet loss rate of rtPS as traffic rate of rtPS changes. We can see that packet loss rate of rtPS for graph coloring algorithm increases as the traffic rate of rtPS changes. Packet loss rate of rtPS for adaptive algorithm and priority algorithm are zero and remain unchanged, this is because that the priority functions take the Qos requirement into account for adaptive algorithm and priority algorithm.

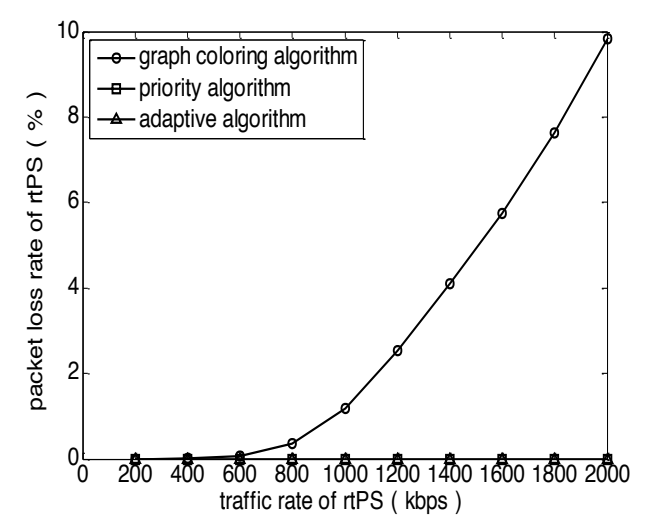

Figure 3. Packet loss rate of rtPS versus traffic rate of rtPS

Fig. 4 demonstrates the variation for throughput of nrtPS as the traffic rate of nrtPS changes. The throughputs of nrtPS for the three algorithms raise when the traffic rate of nrtPS increases. When the traffic rate of nrtPS is small(400kbps-2000kbps), three algorithms have same performance and the throughputs of nrtPS for the three algorithm are equal to the traffic rate of nrtPS. When the traffic rate of nrtPS is large (2400kbps-4000kbps), the graph coloring algorithm has the largest throughput of nrtPS, the reason is that graph coloring algorithm guarantee the QoS of nrtPS at the expense of QoS of rtPS. The throughput of nrtPS for adaptive algorithm is larger than throughput of nrtPS for priority algorithm.

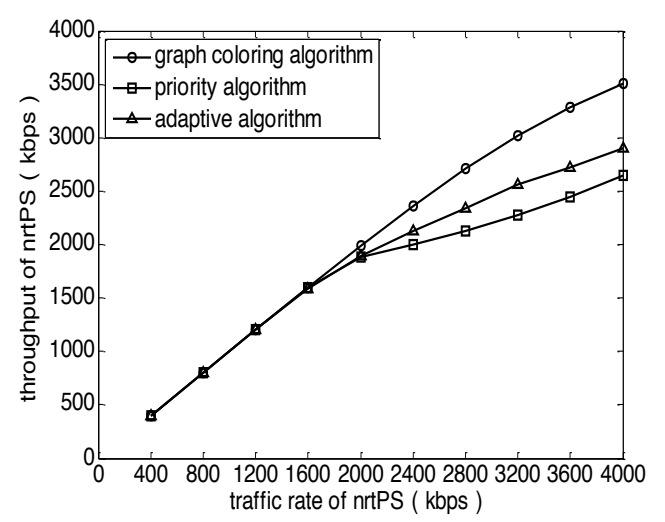

Figure 4. Throughput of nrtPS versus traffic rate of nrtPS

\section{ACKNOWLEDGMENT}

This work was supported by the National Key Technology R\&D Program of China under the grant number 2012BAH16B00, the National Science Foundation for Distinguished Young Scholars of China under the grant number 61003239, the National Science Fund for Distinguished Young Scholars(60725105), National Basic Research Program of China (973 Program)(2009CB320404), Supported by Program for Changjiang Scholars and Innovative Research Team in University(IRT0852).

\section{REFERENCES}

[1] IEEE802.16-2001, "IEEE Standard for Local and Metropolitan Area Networks Part 16: Air Interface for Fixed Broadband Wireless Access Systems," New York, IEEE, 2001.

[2] IEEE802.16d-2004, "IEEE Standard for Local and Metropolitan Area Networks Part 16: Air Interface for Fixed Broadband Wireless Access Systems," New York, IEEE, 2004.

[3] B. Kiernan, R. Murias, I. Kitroser, "IEEE P802.16e/D12: Air Interface for Fixed and Mobile Broadband Wireless Access Systems: Amendment for Physical and Medium Access Control Layers for Combined Fixed and Mobile Operation in Licensed Bands," New York, IEEE, 2005.

[4] Y. Wang, S. Chan, M. Zukerman, "Priority-Based fair Scheduling for Multimedia WiMAX Uplink Traffic," IEEE International Conference on Communications(ICC 2008),2008, PP.301-305. http://dx.doi.org/10.1109/ICC.2008.63

[5] L. F. M Moraes., P. D. J. Maciel, "Analysis and evaluation of a new MAC protocol for broadband wireless access," International conference Wireless Networks, Communications and Mobile Computing, 2005, 1,PP. 107-112.

[6] L. L. Wang, H. M. Xu, "A new management strategy of service flow in IEEE 802.16 systems," IEEE Conference on. Industrial Electronics and Applications, 2008, PP. 1716-1719.

[7] D. Niyato, E. Hossain, "Queue-aware uplink bandwidth allocation for polling services in 802.16 broadband wireless networks," IEEE Global Telecommunications Conference, 2005, 6, PP.5-9.

[8] Q. W. Liu, X. Wang, G. B. Giannakis: A Cross-Layer Scheduling Algorithm With QoS Support in Wireless Networks, IEEE transactions on vehicular technology, 2006, 55(3), PP. 839-847.

[9] L. H. Wan, W. C. Ma, "A Cross-layer Packet Scheduling and Subchannel Allocation Scheme in 802.16e OFDMA System," IEEE WCNC, Hong Kong, China, 2007, PP. 1867-1872.

[10] T. A. Yahiya, A. L. Beylot, G. Pujolle, "Cross-layer Multiservice Scheduling for Mobile WiMAX Systems," IEEE WCNC, Las Vegas, USA, 2008, PP. 1867-1872.

[11] Z. H. Zeng, F. Q. Liu, J. Tao, "Research on MAC Scheduling Mechanism in IEEE 802.16 Mesh mode," Computer Engineering and Applications, 2005, 23, PP.135-138+155.

[12] S. Ramanathan, "A unified framework and algorithm for channel assignment in wireless networks," Wireless Networks, 1999, 5(2), PP. 81-94. 
[13] H. T. Zheng, C. Y. Peng, "Collaboration and fairness in opportunistic spectrum access," IEEE ICC, Seoul, Korea, 2005. $3132-3136$.

\section{AUTHORS}

L. Y. Xiao is with the China Organizational Name Administration Center, Beijing, 100028, China (e-mail: xiaoly@ conac.cn).

R. Wang is with the China Organizational Name Administration Center, Beijing, 100028, China (e-mail: wangrui@,conac.cn).
Z. Wang is with the China Organizational Name Administration Center, Beijing, 100028, China (e-mail: wangzheng@ conac.cn).

H. N. Wang is with the China Organizational Name Administration Center, Beijing, 100028, China (e-mail: wanghn@ conac.cn).

This article is an extended and modified version of a paper presented at the 2013 Chinese Intelligent Automation Conference (CIAC2013), held in Yangzhou, Jiangsu Province, China, in August 2013. Submitted 23 May 2013. Published as re-submitted by the authors 23 July 2013. 